\title{
Purchase of antidepressant agents by patients with type 1 diabetes is associated with increased mortality rates in women but not in men
}

\author{
A. J. Ahola $\cdot$ V. Harjutsalo $~ M$. Saraheimo . \\ C. Forsblom • P.-H. Groop • the FinnDiane Study Group
}

Received: 11 August 2011 /Accepted: 29 September 2011 /Published online: 28 October 2011

(C) Springer-Verlag 2011

\begin{abstract}
Aims/hypothesis Individuals with diabetes have increased mortality rates compared with the general population. In patients with type 2 diabetes depression further contributes to the increased mortality. Depression and mortality rates in patients with type 1 diabetes are an understudied phenomenon. We therefore studied their association in a prospective setting. Methods We followed 4,174 participants (51\% men, age $39 \pm 12$ years, diabetes duration $22 \pm 12$ years [mean \pm
\end{abstract}

Electronic supplementary material The online version of this article (doi:10.1007/s00125-011-2347-6) contains a peer-reviewed but unedited list of participating centres and staff, which is available to authorised users.

A. J. Ahola $\cdot$ V. Harjutsalo $\cdot$ M. Saraheimo $\cdot$ C. Forsblom $\cdot$

P.-H. Groop $(\bowtie)$

Folkhälsan Institute of Genetics, Folkhälsan Research Center, Biomedicum Helsinki, University of Helsinki,

PO Box 63, 00014 Helsinki, Finland

e-mail: per-henrik.groop@helsinki.fi

A. J. Ahola $\cdot$ V. Harjutsalo $\cdot$ M. Saraheimo $~ C$. Forsblom •

P.-H. Groop

Department of Medicine, Division of Nephrology,

Helsinki University Central Hospital,

Helsinki, Finland

\section{A. J. Ahola}

Division of Nutrition, Department of Food and Environmental

Sciences, University of Helsinki,

Helsinki, Finland

\section{Harjutsalo}

National Institute for Health and Welfare,

Diabetes Prevention Unit,

Helsinki, Finland

P.-H. Groop

Baker IDI Heart and Diabetes Institute,

Melbourne, VIC, Australia
SD]) in the Finnish Diabetic Nephropathy Study (FinnDiane) for an average of 9 years. Depression was defined as purchase of antidepressant agents at baseline and during follow-up. These data were obtained from the Finnish Drug Prescription Register. Data on allcause mortality and cause of death were obtained from the Finnish Cause of Death Register.

Results At baseline, 313 (7.5\%) patients had purchased antidepressant agents. During follow-up 758 (18.2\%) additional cases were observed. Purchasers of antidepressant agents at baseline had the highest 10-year cumulative mortality rate $(22.5 \%$ [95\% CI $18.1,26.6])$, followed by those with such purchases during follow-up $(18.0 \%[15.4,20.5])$ and those with no purchases $(10.1 \%[9.0,11.2], p<0.001)$. In the adjusted Cox regression models (age, diabetes duration, diastolic blood pressure, smoking, $\mathrm{HbA}_{1 \mathrm{c}}$ and nephropathy), the purchase of antidepressant agents at baseline was associated with mortality in women, but not in men. Cardiovascular diseases were the major cause of death in non-purchasers of antidepressant agents. In antidepressant purchasers, chronic diabetic complications were the most frequent underlying cause of death.

Conclusions/interpretation In a population of patients with type 1 diabetes, purchase of antidepressant agents was associated with increased mortality rates in women, but not in men.

Keywords Cause of death - Depression - Mortality - Type 1 diabetes

$\begin{array}{ll}\text { Abbreviations } \\ \text { CVD } & \text { Cardiovascular disease } \\ \text { DPR } & \text { Drug Prescription Register } \\ \text { ESRD } & \text { End-stage renal disease } \\ \text { FinnDiane } & \text { Finnish Diabetic Nephropathy Study } \\ \text { IQR } & \text { Interquartile range }\end{array}$




\section{Introduction}

Several studies have shown higher mortality rates in individuals with diabetes as opposed to those without [1-3]. For example, in a Finnish population of patients with type 1 diabetes, a 3.7 times higher mortality rate than in the general population was observed [2]. Diabetes, among other causes, significantly contributes to premature death related to cardiovascular [3] and kidney diseases [4].

Depression is highly prevalent among patients with diabetes. In a recent meta-analysis, clinical depression was observed in $12.0 \%$ of participants with type 1 diabetes and in $3.2 \%$ of healthy controls [5]. Comorbid depression in diabetes may have critical consequences in the form of poor adherence to diet, exercise, glucose monitoring and medication regimen $[6,7]$. In addition to its effects on self-care adherence, depression has also been associated with deleterious physiological changes, such as dysregulation of the hypothalamic-pituitary axis, downregulation of cellular and humoral responses, and changes in the sympathetic nervous system [8, 9]. Poor self-care behaviour and physiological dysregulation may jointly or separately have an adverse influence on diabetes outcomes. Indeed, patients with comorbid depression have shown an increased risk of various diabetic complications [10].

Importantly, many studies have reported an association between depression and increased mortality rates in the general population [11] and in patients with type 2 diabetes [12-14]. Despite ample research, to our knowledge, no study on depression and mortality rates in patients with type 1 diabetes has been published. We therefore studied the effects of depression on all-cause and cause-specific mortality in a population of patients with type 1 diabetes. Based on previous observations, we expected to find higher all-cause mortality rates in individuals with depression than in non-depressed patients.

\section{Methods}

Study design The Finnish Diabetic Nephropathy Study (FinnDiane) is a large national multicentre study seeking to identify factors associated with diabetic complications. Since its launch in 1997, data from more than 4,800 adult patients with type 1 diabetes have been collected. Included in the current analyses are all patients who were recruited prior to the end of 2009 and have given consent to link their data with data in the Drug Prescription Register (DPR). Thus, data from a total of 4,174 patients $(51 \%$ men, age at baseline $39 \pm 12$ years, diabetes duration $22 \pm 12$ years $[$ mean $\pm \mathrm{SD}])$ were included. Type 1 diabetes was defined as onset of diabetes before the age of 35 years, permanent insulin treatment initiated within 1 year of diagnosis and C- peptide negativity. The study protocol was approved by the Ethics Committee of Helsinki University Central Hospital and written informed consent was obtained prior to participation.

Determination of participant characteristics During the baseline visit, the patients' height and weight were measured in light clothing and BMI was calculated. Waist circumference was measured at the midpoint between the lowest rib and the iliac crest. Blood pressure was measured in a sitting position after a $10 \mathrm{~min}$ rest. The mean of two blood pressure measurements, performed 2 min apart, was calculated. $\mathrm{HbA}_{1 \mathrm{c}}$ was determined locally using standardised assays. Self-reported data for smoking and social class (grouped as unskilled/skilled blue collar, upper/lower white collar, farmers and others) were collected. Unskilled blue collar workers were classified as having a low socioeconomic status. Finally, renal status was assessed by AER in at least two of three timed $24 \mathrm{~h}$ or overnight urine collections. Patients were classified as having normal AER $(<20 \mu \mathrm{g} / \mathrm{min}$ or $<30 \mathrm{mg} / 24 \mathrm{~h})$, microalbuminuria (AER $\geq 20$ and $<200 \mu \mathrm{g} / \mathrm{min}$, or $\geq 30$ and $<300 \mathrm{mg} / 24 \mathrm{~h}$ ), macroalbuminuria (AER $\geq 200 \mu \mathrm{g} / \mathrm{min}$ or $\geq 300 \mathrm{mg} / 24 \mathrm{~h}$ ) or end-stage renal disease (ESRD) (undergoing dialysis or having had a kidney transplant). Diabetic nephropathy was defined as macroalbuminuria or ESRD.

Data on antidepressant medication purchase Data from the DPR of the Social Insurance Institute of Finland were used to identify purchases of antidepressant agents by linking the FinnDiane data using the patient's unique identification number. Since late 1994, all prescriptions in outpatient care have been included in the DPR, which contains complete information on all prescribed, purchased and reimbursed medications. From the register, we searched for all drugs in the classes N06A and N06CA (Anatomical Therapeutic Chemical Classification System with Defined Daily Doses [15]). For the analyses, depression at baseline was defined as purchase of any such medication within 1 year prior to the baseline visit. Patients who had not purchased antidepressant agents at baseline were further divided into those without any such purchase during the study and those who purchased during the follow-up.

Mortality data At the end of 2009, data on all-cause mortality were obtained from the Finnish Cause of Death Register. These data were used to calculate the total mortality rate in the study population. In Finland, data on causes of deaths are retrospectively updated once a year; thus for this study, cause of death data were available for all deaths up to the end of 2008. In this register, the underlying cause of death is reported on the basis of the coroner's 
evaluation. Cardiovascular deaths included all deaths due to diseases of the circulatory system (International Statistical Classification of Diseases and Related Health Problems codes I00-199). The majority of these deaths were due to ischaemic heart diseases (I20-I25) and cerebrovascular diseases (I60-I69).

Statistical analyses Descriptive statistics are reported as percentages for categorical data, mean $\pm \mathrm{SD}$ for normally distributed data and median (interquartile range, IQR) for non-normally distributed data. The $\chi^{2}$ test, one-way ANOVA, independent-sample $t$ test, and Kruskal-Wallis and Mann-Whitney $U$ tests were used to compare the groups, as appropriate. The cumulative mortality rate was assessed using the Kaplan-Meier method. Multivariate associations between the purchase of antidepressant agents and mortality were studied using the Cox proportional hazards model. The effect modifications were controlled for by adding interaction terms to the model. A value of $p<0.05$ was considered statistically significant. Data were analysed using SAS statistical software, version 9.2 (SAS, Cary, NC, USA) and SPSS 17.0 for Windows (SPSS, Chicago, IL, USA).

\section{Results}

We followed 4,174 patients for an average of 9 years (range 0-16 years). Of the three groups formed, those without any purchases of antidepressant agents were most frequently male, and had the shortest duration of diabetes, the lowest frequency of nephropathy and the lowest $\mathrm{HbA}_{1 \mathrm{c}}$ levels (Table 1).

In all, $313(7.5 \%)$ of the patients studied had purchased antidepressant agents at baseline and a further 758 (18.2\%) individuals made such purchases during follow-up. During follow-up, 474 (11.4\%) deaths occurred. Of antidepressant agent purchasers, $19.8 \%$ of those who had purchased at baseline and $16.2 \%$ of purchasers during follow-up died. Among non-purchasers of antidepressant agents, 9.3\% died during the follow-up $(p<0.001$ for comparison of the three groups). The 10-year cumulative mortality rate was highest among purchasers of antidepressant agents at baseline, followed by purchasers during follow-up and finally nonpurchasers $(22.5 \%$ [95\% CI 18.1, 26.6], 18.0\% [15.4, $20.5 \%]$ and $10.1 \%$ [9.0, 11.2], respectively, $p<0.001$ logrank test). Separate analyses for men and women gave similar results (data not shown).

Table 1 Patient characteristics

\begin{tabular}{|c|c|c|c|c|}
\hline \multirow{2}{*}{$\begin{array}{l}\text { Characteristic } \\
n(\%)\end{array}$} & \multicolumn{3}{|c|}{ Antidepressant agent purchase } & \multirow[t]{2}{*}{$p$ value } \\
\hline & $\begin{array}{l}\text { At baseline } \\
313(7.5)\end{array}$ & $\begin{array}{l}\text { During follow-up } \\
758(18.2)\end{array}$ & $\begin{array}{l}\text { No purchase } \\
3103(74.3)\end{array}$ & \\
\hline \multicolumn{5}{|c|}{ Types of antidepressants used } \\
\hline SSRI & 53.7 & 63.9 & NA & \\
\hline Tricyclic medication & 25.9 & 15.6 & NA & \\
\hline Other & 20.4 & 20.6 & NA & $<0.001$ \\
\hline Male sex $(\%)$ & 39.3 & 45.8 & $54.1^{\mathrm{bc}}$ & $<0.001$ \\
\hline Age (years) & $43.4 \pm 11.4$ & $39.9 \pm 11.5^{\mathrm{b}}$ & $38.8 \pm 12.4^{\mathrm{b}}$ & $<0.001$ \\
\hline Diabetes duration (years) & $26.2 \pm 12.1$ & $24.2 \pm 11.5^{\mathrm{d}}$ & $21.3 \pm 12.4^{\mathrm{bc}}$ & $<0.001$ \\
\hline Nephropathy (\%) & 30.3 & 29.8 & $20.5^{\mathrm{bc}}$ & $<0.001$ \\
\hline $\mathrm{HbA}_{1 \mathrm{c}}(\%)$ & $8.5(7.6-9.6)$ & $8.5(7.6-9.3)$ & $8.2(7.4-9.2)^{\mathrm{be}}$ & $<0.001$ \\
\hline $\mathrm{HbA}_{1 \mathrm{c}}(\mathrm{mmol} / \mathrm{mol})$ & $69.4(59.6-81.4)$ & $69.4(59.6-78.1)$ & $66.1(57.4-77.0)$ & $<0.001$ \\
\hline BMI $\left(\mathrm{kg} / \mathrm{m}^{2}\right)$ & $25.1(22.7-27.4)$ & $24.8(22.4-27.2)$ & $24.7(22.7-26.9)$ & 0.606 \\
\hline Waist circumference $(\mathrm{cm})$ & $86(77-94)$ & $85(77-93)$ & $85(78-93)$ & 0.602 \\
\hline Systolic BP (mmHg) & $133(122-146)$ & $132(121-146)$ & $132(121-145)$ & 0.594 \\
\hline Diastolic BP (mmHg) & $79(72-86)$ & $80(74-87)$ & $80(72-86)$ & 0.072 \\
\hline Current smoking (\%) & 26.6 & 27.7 & $22.0^{\mathrm{f}}$ & 0.002 \\
\hline Low SES (\%) & 19.4 & 21.3 & 19.5 & 0.540 \\
\hline
\end{tabular}

Data are presented as frequency (\%) for categorical variables, and mean $\pm \mathrm{SD}$ or median (IQR) for continuous variables

${ }^{\mathrm{a}} p$ values are for comparisons among the three groups (for the types of medication used, only two groups were compared); ${ }^{\mathrm{b}} p<0.001$ compared with those with purchases of antidepressant agents at baseline; ${ }^{c} p<0.001$ compared with those with purchases of antidepressant agents during follow-up; ${ }^{\mathrm{d}} p<0.05$ compared with those with purchases of antidepressant agents at baseline; ${ }^{\mathrm{e}} p<0.01$ compared with those with purchases of antidepressant agents at baseline; ${ }^{\mathrm{f}} p<0.01$ compared with those with purchases of antidepressant agents during follow-up

SES, socioeconomic status; SSRI, selective serotonin reuptake inhibitors 
The interaction term between sex and antidepressant purchase status was not significant, thus the effect of depression on mortality rates was similar in men and women. However, as nephropathy and its risk factors, such as $\mathrm{HbA}_{1 \mathrm{c}}$, are major contributing factors to mortality, they were included in the multivariate models. The effects of these variables on mortality rates were different in men and women $(p<0.001$ for interaction between sex and nephropathy, $p<0.01$ for interaction between sex and $\mathrm{HbA}_{1 \mathrm{c}}$ ), and therefore the results are presented separately. In men and women, adjusted for age, diabetes duration, diastolic blood pressure and smoking, the two groups of antidepressant agent purchasers had higher HRs of death during follow-up, relative to non-purchasers (Table 2). After further adjustment with $\mathrm{HbA}_{1 \mathrm{c}}$, the HRs remained significant in women, but not men. In the final model, which also included nephropathy status, the HR was significantly higher for women who had purchased antidepressant agents at baseline. No differences in the HRs of death were observed in patients using different kinds of antidepressant agents (selective serotonin reuptake inhibitors, tricyclic medication and other types of medication). Figure 1 shows the cumulative mortality curves based on the final Cox proportional hazards model for men (Fig. 1a) and women (Fig. 1b).

Cause of death data was available for 455 deaths (Table 3). Cardiovascular disease (CVD) was the underlying cause of death in $31 \%$ of patients who had purchased antidepressant agents at baseline, $33 \%$ of purchasers during the follow-up and $45 \%$ of non-purchasers, respectively ( $p<$ $0.05)$. The three groups also differed with regard to the frequency of deaths due to chronic diabetic complications, which was highest in individuals who purchased antidepressant agents during follow-up (46\%) and lowest among non-purchasers $(31 \% ; p<0.05$ for comparison of three groups). Acute diabetic complications were the underlying cause of death in $10 \%$ of deaths in antidepressant agent purchasers at baseline. Although a non-significant difference, this was double the rate observed both in individuals who purchased antidepressant agents during follow-up and in non-purchasers. There were six suicides, two in each of the three groups.

\section{Discussion}

In the current study of patients with type 1 diabetes, individuals who had purchased antidepressant agents (considered to be 'depressed') had higher 10 year cumulative mortality rates than non-purchasers. These results are in concordance with many of the previous studies conducted in patients with diabetes. During an 8 year period in the National Health and Nutrition Examination Survey (NHANES) I Epidemiologic Follow-up Study, individuals with concomitant diabetes and depression had increased odds of all-cause mortality compared with those with diabetes only [16]. In another study among 10,704 Medicare beneficiaries with diabetes, those with depression had a 36-38\% increased risk of all-cause mortality over a 2 year period compared with those without depression [13]. In a third study of 4,184 patients with diabetes, baseline major depression, relative to no depression, was also significantly associated with allcause mortality [14]. Unlike our study, all the abovementioned studies included, exclusively or predominantly, patients with type 2 diabetes. Thus far, the association between depression and mortality rates in patients with type 1 diabetes has not been explored. The two types of diabetes, however, differ substantially in their aetiologies and management, and therefore should be considered as two distinct entities.

Table 2 Hazard ratios of death from all causes according to purchase of antidepressant agents

\begin{tabular}{|c|c|c|c|c|}
\hline Antidepressant agent purchase by sex & Model 1 & Model 2 & Model 3 & Model 4 \\
\hline \multicolumn{5}{|l|}{ Men } \\
\hline None & Reference & Reference & Reference & Reference \\
\hline At baseline & $1.65(1.12,2.42)$ & $1.55(1.03,2.35)$ & $1.28(0.83,1.99)$ & $1.12(0.71,1.77)$ \\
\hline During follow-up & $1.46(1.11,1.93)$ & $1.39(1.04,1.85)$ & $1.32(0.98,1.77)$ & $1.17(0.87,1.58)$ \\
\hline \multicolumn{5}{|l|}{ Women } \\
\hline None & Reference & Reference & Reference & Reference \\
\hline At baseline & $2.00(1.34,3.00)$ & $1.99(1.29,3.07)$ & $1.94(1.25,3.02)$ & $2.15(1.34,3.45)$ \\
\hline During follow-up & $1.78(1.27,2.49)$ & $1.70(1.18,2.43)$ & $1.75(1.22,2.52)$ & $1.41(0.96,2.06)$ \\
\hline
\end{tabular}

Data are presented as HR $(95 \% \mathrm{CI})$

Model 1: adjusted for age and diabetes duration

Model 2: adjusted for age, diabetes duration, diastolic BP and smoking

Model 3: adjusted for age, diabetes duration, diastolic BP, smoking and $\mathrm{HbA}_{1 \mathrm{c}}$

Model 4: adjusted for age, diabetes duration, diastolic BP, smoking, $\mathrm{HbA}_{1 \mathrm{c}}$ and nephropathy 
Fig. 1 Cumulative survival according to the purchase of antidepressant agents in (a) men $(p=0.330)$ and $(\mathbf{b})$ women $(p=0.008)$. Black line, no purchases; dashed line, purchase during follow-up; dotted line, purchase at baseline

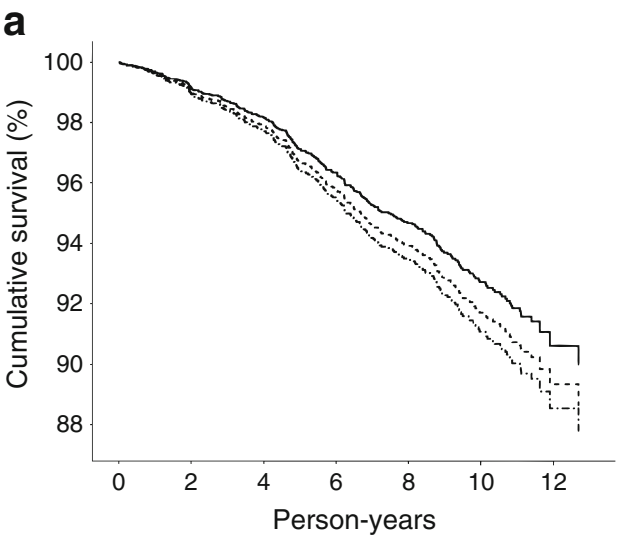

Separate analyses for men and women in the present study showed clear differences in depression-related survival rates between the sexes. In these analyses, depression significantly contributed to reduced survival during follow-up in women, but not in men. This finding may be important as depression is more frequently observed in women [17]. The reason why women seem more vulnerable to the effects of depression is not known. Brodaty et al. found that major depression in women, but not in men increases mortality rates compared with the general population [18]. However, the exclusion of suicides in that study abolished the effect. Here, the exclusion of participants who committed suicide from the analyses did not change the results (data not shown). Moreover, a number of studies have reported an increased risk of mortality in depressed men, but not in women [19, 20]. A meta-analysis including studies with random samples from different communities found that the relative mortality risk for depressed men was only non-significantly higher than for depressed women [11]. If the current results can be verified, the underlying reasons for the observed sex-related difference should be investigated in the future.

We observed some differences in mortality rates between antidepressant agent purchasers at the time of baseline visit b

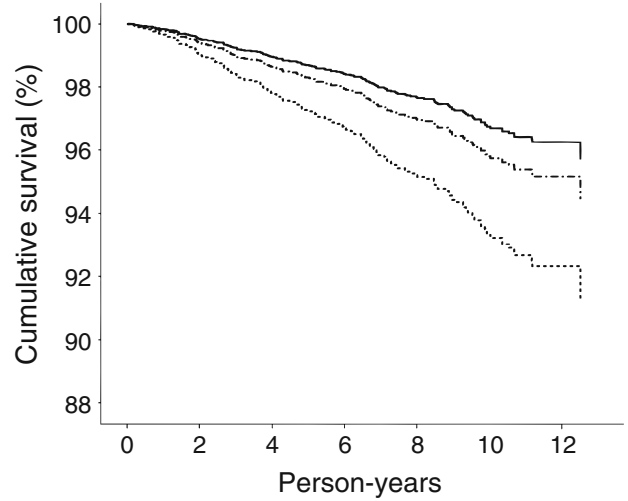

and those who purchased during follow-up. The exact reason for this difference is not known, but several explanations seem plausible. First, depression present at baseline may be more long-standing and thus have more time to contribute to poor outcomes. Second, some changes in the prescription of antidepressant agents may have taken place over the years. While earlier such medication may have been prescribed primarily for more severe depression, prescription practices may have changed to include less severe cases. Thus, purchasers of antidepressant agents at baseline would, on average, include those with more severe depression, thus leading to a heightened risk of increased mortality.

Cardiovascular deaths accounted for most of the deaths in the current study population, followed by chronic diabetic complications. In Finland, CVDs are the leading underlying cause of death among elderly individuals [21]. However, in individuals of working age, alcohol-related deaths play a leading role. While it is probable that individuals with high alcohol intake are under-represented in our study, our results are in concordance with previous findings on the heightened risk of CVD and on CVDrelated deaths in patients with diabetes [22, 23]. Similarly,

Table 3 Causes of death by purchase of antidepressant agents

\begin{tabular}{|c|c|c|c|c|c|}
\hline \multirow{2}{*}{$\begin{array}{l}\text { Deaths } \\
\text { Cause } \\
n\end{array}$} & \multicolumn{4}{|c|}{ Antidepressant agent purchase } & \multirow[t]{2}{*}{$p$ value } \\
\hline & Percentage of all & $\begin{array}{l}\text { At baseline } \\
59\end{array}$ & $\begin{array}{l}\text { During follow-up } \\
116\end{array}$ & $\begin{array}{l}\text { No purchases } \\
280\end{array}$ & \\
\hline Cardiovascular diseases & 40.0 & $18(30.5)$ & $38(32.8)$ & $126(45.0)$ & 0.022 \\
\hline Chronic diabetic complications & 35.8 & $22(37.3)$ & $53(45.7)$ & $88(31.4)$ & 0.026 \\
\hline Cancers & 7.7 & $5(8.5)$ & $7(6.0)$ & $23(8.2)$ & 0.738 \\
\hline Acute diabetic complications & 5.9 & $6(10.2)$ & $6(5.2)$ & $15(5.4)$ & 0.336 \\
\hline Alcohol-related & 1.3 & $1(1.7)$ & $2(1.7)$ & $3(1.1)$ & 0.843 \\
\hline Suicide & 1.3 & $2(3.4)$ & $2(1.7)$ & $2(0.7)$ & 0.237 \\
\hline Other & 7.9 & $5(8.5)$ & $8(6.9)$ & $23(8.2)$ & 0.894 \\
\hline
\end{tabular}

Data are presented as $n(\%)$

${ }^{\mathrm{a}} p$ values are for comparisons among the three groups 
in a cross-sectional Look Action for Health in Diabetes (AHEAD) study, symptoms of depression or use of antidepressant agents were associated with a range of CVD risk factors, including hypertension, smoking and obesity [24]. In the current study, patients without any purchases of antidepressant agents died most frequently of CVD-related causes. However, chronic diabetic complications were the most common cause of death among purchasers of antidepressant agents. It is probable that the onset of chronic complications in diabetes triggers or aggravates depression. However, established depression may also contribute to the development of complications. Interestingly, $10 \%$ of antidepressant agent purchasers at baseline died of acute diabetic complications. Although not significant, this rate was twice as high as in the other patient groups. The question of whether acute diabetic complications contribute to increased mortality rates in these patients needs to be investigated in a larger cohort.

The current study has several strengths and limitations. First, the FinnDiane study comprises a fairly large proportion of all Finnish patients with type 1 diabetes, and although the study is not population-based, the distribution of participants follows that of the general population in Finland. However, some selection bias favouring those with better mental and general health is possible. Rather than strengthening our results, this selection bias is most likely to have somewhat diluted them. Second, the population of 4,174 thoroughly examined individuals in this study offers a reasonable basis to investigate the association between depression and mortality. Another major strength is that, instead of relying on selfreported data, data on the main study variables were based on actual diagnoses or on register data. This diminishes the potential misclassification of participants, for example, with regard to diabetes and causes of deaths. Sourcing of data on medication use from the DPR is particularly valuable, as selfreported use of antidepressant agents may be prone to underreporting. At the same time, depression classification on the basis of the register data may also be problematic. First, not all depressed patients seek professional help. Thus in the Health 2000 Study, which was conducted in the Finnish general population, only one third of individuals with major depressive disorder reported currently receiving antidepressants or psychological treatment [25]. Moreover, in that study, the use of antidepressants was associated with female sex. Second, data on indications are not currently available from the register. This is important as some antidepressant agents may also be used to manage neuropathic pain. Depression is, however, commonly associated with chronic pain, making it difficult to distinguish these two phenomena [26]. Unfortunately, data on neuropathy status were not available for the current analyses, and results therefore need to be interpreted in light of this limitation.
In conclusion, the 10 year cumulative mortality rate was highest in patients who had purchased antidepressant agents at baseline, followed by those purchasing these agents during the follow-up period and finally those who made no such purchases. During an average follow-up of 9 years, the purchase of antidepressant agents at baseline, in multivariate models, was associated with increased mortality rates in women, but not in men. Relative to individuals without any purchases of antidepressant agents, purchasers had a greater frequency of chronic diabetic complications as underlying cause of death.

Acknowledgements This study was supported by grants from the Signe and Ane Gyllenberg Foundation, Folkhälsan Research Foundation, and Wilhelm and Else Stockmann Foundation. The skilled technical assistance of A. Sandelin and J. Tuomikangas is gratefully acknowledged. The authors acknowledge all the physicians and nurses at each centre participating in the collection of patients (see ESM).

Contribution statement All the authors contributed to the conception and design of the study, interpretation of data and critical revision of the manuscript for important intellectual content, and gave final approval of the version to be published. AJA and VH contributed to analysis and drafting of the manuscript.

Duality of interest The authors declare that there is no duality of interest associated with this manuscript.

\section{References}

1. Gu K, Cowie CC, Harris MI (1998) Mortality in adults with and without diabetes in a national cohort of the U.S. population, 1971-1993. Diabetes Care 21:1138-1145

2. Asao K, Sarti C, Forsen T et al (2003) Long-term mortality in nationwide cohorts of childhood-onset type 1 diabetes in Japan and Finland. Diabetes Care 26:2037-2042

3. Barnett KN, Ogston SA, McMurdo ME, Morris AD, Evans JM (2010) A 12-year follow-up study of all-cause and cardiovascular mortality among 10,532 people newly diagnosed with type 2 diabetes in Tayside, Scotland. Diabet Med 27:1124-1129

4. Groop PH, Thomas MC, Moran JL et al (2009) The presence and severity of chronic kidney disease predicts all-cause mortality in type 1 diabetes. Diabetes 58:1651-1658

5. Barnard KD, Skinner TC, Peveler R (2006) The prevalence of comorbid depression in adults with type 1 diabetes: systematic literature review. Diabet Med 23:445-448

6. Gonzalez JS, Peyrot M, McCarl et al (2008) Depression and diabetes treatment nonadherence: a meta-analysis. Diabetes Care 31:2398-2403

7. Lin EH, Katon W, von Korff M et al (2004) Relationship of depression and diabetes self-care, medication adherence, and preventive care. Diabetes Care 27:2154-2160

8. Joynt KE, Whellan DJ, O'Connor CM (2003) Depression and cardiovascular disease: mechanisms of interaction. Biol Psychiatry $54: 248-261$

9. Kiecolt-Glaser JK, Glaser R (2002) Depression and immune function: central pathways to morbidity and mortality. J Psychosom Res 53:873-876 
10. de Groot M, Anderson R, Freedland KE, Clouse RE, Lustman PJ (2001) Association of depression and diabetes complications: a meta-analysis. Psychosom Med 63:619-630

11. Cuijpers P, Smit F (2002) Excess mortality in depression: a metaanalysis of community studies. J Affect Disord 72:227-236

12. Zhang X, Norris SL, Gregg EW, Cheng YJ, Beckles G, Kahn HS (2005) Depressive symptoms and mortality among persons with and without diabetes. Am J Epidemiol 161:652-660

13. Katon W, Fan MY, Unutzer J, Taylor J, Pincus H, Schoenbaum M (2008) Depression and diabetes: a potentially lethal combination. J Gen Intern Med 23:1571-1575

14. Lin EH, Heckbert SR, Rutter CM et al (2009) Depression and increased mortality in diabetes: unexpected causes of death. Ann Fam Med 7:414-421

15. WHO Collaborating Centre for Statistics Methodology. ATC/ DDD Index 2011. Available from www.whocc.no/atcddd/. Accessed 5 October 2011.

16. Egede LE, Nietert PJ, Zheng D (2005) Depression and all-cause and coronary heart disease mortality among adults with and without diabetes. Diabetes Care 28:1339-1345

17. Grigoriadis S, Robinson GE (2007) Gender issues in depression. Ann Clin Psychiatry 19:247-255

18. Brodaty H, MacCuspie-Moore CM, Tickle L, Luscombe G (1997) Depression, diagnostic sub-type and death: a 25 year follow-up study. J Affect Disord 46:233-242
19. Ryan J, Carriere I, Ritchie K et al (2008) Late-life depression and mortality: influence of gender and antidepressant use. $\mathrm{Br} \mathrm{J}$ Psychiatry 192:12-18

20. Sun W, Schooling CM, Chan WM, Ho KS, Lam TH (2011) The association between depressive symptoms and mortality among Chinese elderly: a Hong Kong cohort study. J Gerontol A Biol Sci Med Sci 66:459-466

21. Official Statistic of Finland (OSF): Causes of death (e-publication)]. ISSN=1799-5078. Helsinki: Statistic Finland. Available from: www. tilastokeskus.fi/til/ksyyt/index_en.html. Accessed 30 May 2011.

22. Kannel WB, McGee DL (1979) Diabetes and cardiovascular disease. The Framingham study. JAMA 241:2035-2038

23. Laing SP, Swerdlow AJ, Slater SD et al (2003) Mortality from heart disease in a cohort of 23,000 patients with insulin-treated diabetes. Diabetologia 46:760-765

24. Rubin RR, Gaussoin SA, Peyrot M et al (2010) Cardiovascular disease risk factors, depression symptoms and antidepressant medicine use in the Look AHEAD (Action for Health in Diabetes) clinical trial of weight loss in diabetes. Diabetologia 53:1581-1589

25. Hämäläinen J, Isometsä E, Sihvo S, Kiviruusu O, Pirkola S, Lönnqvist J (2009) Treatment of major depressive disorder in the Finnish general population. Depress Anxiety 26:1049-1059

26. Fishbain DA, Cutler R, Rosomoff HL, Rosomoff RS (1997) Chronic pain-associated depression: antecedent or consequence of chronic pain? A review. Clin J Pain 13:116-137 